\title{
LIPSCHITZ EQUIVALENCE OF A CLASS OF SELF-SIMILAR SETS
}

\author{
Xiu Chen, Kan Jiang* and Wenxia Li
}

East China Normal University, Shanghai Key Laboratory of PMMP, Department of Mathematics Shanghai 200241, P. R. China; chenxiu1216@163.com

Utrecht University, Fac Wiskunde en informatica and MRI, Department of Mathematics Budapestlaan 6, P.O. Box 80.000, 3508 TA Utrecht, The Netherlands; K.Jiang1@uu.nl

East China Normal University, Shanghai Key Laboratory of PMMP, Department of Mathematics Shanghai 200241, P. R. China; wxli@math.ecnu.edu.cn

\begin{abstract}
We consider a class of homogeneous self-similar sets with complete overlaps and give a sufficient condition for the Lipschitz equivalence between members in this class.
\end{abstract}

\section{Introduction}

Let $\left(X_{i}, d_{i}\right), i=1,2$, be metric spaces. For nonempty sets $A_{i} \subseteq X_{i}$ we say they are Lipschitz equivalent, denoted by $A_{1} \simeq A_{2}$, if there exists a bijection $\phi: A_{1} \rightarrow A_{2}$ and a constant $c>0$ such that

$$
c^{-1} d_{1}(x, y) \leq d_{2}(\phi(x), \phi(y)) \leq c d_{1}(x, y) \text { for any } x, y \in A_{1} .
$$

Lipschitz equivalence can be used to classify fractal sets. Since late 80's many works have been devoted to the study of Lipschitz equivalence (see $[2,3,4,5,7,8,9,11$, $13,14,15,16]$ and references therein). An effective method, to our knowledge, was first employed in [11] for establishing a bi-Lipschitz mapping between the $\{1,4,5\}$ Cantor set and the $\{1,3,5\}$-Cantor set, the main idea of which is to show these two self-similar sets to have the same graph-directed structure satisfying the strong separation condition. A sufficient condition was given in [1, Theorem 2.11] to judge whether or not a self-similar set has a graph-directed structure satisfying the open set condition or even the strong separation condition.

In the present paper we consider the homogeneous iterated function system (IFS) $\left\{f_{i}(x)=\lambda x+a_{i}: 1 \leq i \leq m\right\}$ where $x, a_{i} \in \mathbf{R}, \lambda \in(0,1)$ and the integer $m \geq 3$. For a vector $\left(k_{1}, \ldots, k_{n}\right)$ of integers with $k_{1}>k_{2}>\cdots>k_{n} \geq 2$, let $\mathbf{A}_{k_{1}, \ldots, k_{n}}$ be the collection of translations $\mathbf{a}=\left(a_{1}, a_{2}, \cdots, a_{m}\right)$ satisfying the following conditions (I) (II) and (III):

(I) $0=a_{1}<a_{2}<\cdots<a_{m}=1-\lambda$;

(II) Any three intervals in $\left\{f_{i}([0,1]): 1 \leq i \leq m\right\}$ do not intersect. $\mid f_{i}([0,1]) \cap$ $f_{j}([0,1]) \mid \in\left\{\lambda^{k_{1}}, \cdots, \lambda^{k_{n}}\right\}$ whenever $f_{i}([0,1]) \cap f_{j}([0,1]) \neq \emptyset$ with $i<j$, where by $|J|$ we denote the length of an interval $J$;

(III) Either $f_{1}([0,1]) \cap f_{j}([0,1])=\emptyset$ for all $j>1$, or $f_{m}([0,1]) \cap f_{j}([0,1])=\emptyset$ for all $j<m$.

From (I) and (II) it follows that when $\left|f_{i}([0,1]) \cap f_{j}([0,1])\right|=\lambda^{k_{\ell}}$ with $i<j$, then $j=i+1$ and $f_{i} \circ f_{m}^{k_{\ell}-1}=f_{j} \circ f_{1}^{k_{\ell}-1}$. Throughout this paper, $f^{i}$ stands for the $i$-th iteration of map $f$ for $i \in \mathbf{N}$. In particular, $f^{0}$ stands for the identity.

https://doi.org/10.5186/aasfm.2017.4238

2010 Mathematics Subject Classification: Primary 28A80, 28A78.

Key words: Complete overlap, homogeneous self-similar sets, Lipschitz equivalence.

* The corresponding author. 
For a translation $\mathbf{a}=\left(a_{1}, a_{2}, \cdots, a_{m}\right) \in \mathbf{A}_{k_{1}, \ldots, k_{n}}$, let

$$
\gamma_{\ell}(\mathbf{a})=\left\{1 \leq i \leq m:\left|f_{i}([0,1]) \cap f_{i+1}([0,1])\right|=\lambda^{k_{\ell}}\right\} \quad \text { for } 1 \leq \ell \leq n .
$$

It is well known that for each $\mathbf{a}=\left(a_{1}, a_{2}, \cdots, a_{m}\right) \in \mathbf{A}_{k_{1}, \ldots, k_{n}}$, there exists a unique nonempty compact set $K_{\mathbf{a}}$ such that $K_{\mathbf{a}}=\bigcup_{1<i<m} f_{i}\left(K_{\mathbf{a}}\right)$ (see [6]). The set $K_{\mathbf{a}}$ is called the self-similar set generated by the IFS $\left\{f_{i}(x)=\lambda x+a_{i}: 1 \leq i \leq m\right\}$. Let $\# S$ denote the number of elements of $S$. In this paper we obtain

Theorem 1.1. For $\mathbf{a}, \mathbf{b} \in \mathbf{A}_{k_{1}, \ldots, k_{n}}$ we have $K_{\mathbf{a}} \simeq K_{\mathbf{b}}$ if $\# \gamma_{\ell}(\mathbf{a})=\# \gamma_{\ell}(\mathbf{b})$ for $1 \leq \ell \leq n$.

It is clear that $\operatorname{dim}_{H} E=\operatorname{dim}_{H} F$ if $E \simeq F$. Thus we have

Corollary 1.2. For $\mathbf{a}, \mathbf{b} \in \mathbf{A}_{k_{1}, \cdots, k_{n}}$ we have $\operatorname{dim}_{H} K_{\mathbf{a}}=\operatorname{dim}_{H} K_{\mathbf{b}}$ if $\# \gamma_{\ell}(\mathbf{a})=$ $\# \gamma_{\ell}(\mathbf{b})$ for $1 \leq \ell \leq n$.

We prove Theorem 1.1 and give some examples in the next section.

\section{Proof of Theorem 1.1}

Before proving Theorem 1.1, let us recall the graph-directed self-similar set (see $[10])$. Let $\mathcal{G}=(V, E)$ be a directed graph where $V$ is a finite set of vertexes and $E$ is a finite set of directed edges. Assume that for any $u \in V$ there is at least one edge in $E$ starting from $u$. For an $e \in E$, let $f_{e}: \mathbf{R}^{n} \rightarrow \mathbf{R}^{n}$ be a similitude with ratio $\rho_{e} \in(0,1)$, namely

$$
\left|f_{e}(x)-f_{e}(y)\right|=\rho_{e}|x-y| \text { for any } x, y \in \mathbf{R}^{n} .
$$

Then there exist unique nonempty compact sets $\left\{F_{u}: u \in V\right\}$ such that

$$
F_{u}=\bigcup_{v \in V} \bigcup_{e \in E_{u, v}} f_{e}\left(F_{v}\right) \text { for all } u \in V,
$$

where $E_{u, v}$ is the set of directed edges starting from $u$ and ending at $v$. The compact sets $\left\{F_{u}: u \in V\right\}$ in (1) is called the graph-directed self-similar sets generated by $\left\{V, E,\left\{f_{e}: e \in E\right\}\right\}$. In addition, $\left\{F_{u}: u \in V\right\}$ is said to satisfy the strong separation condition if the sets in the right side of (1) are pairwise disjoint. An easy-to-prove result on the Lipschitz equivalence between two graph-directed self-similar sets is as follows (also see [11]).

Lemma 2.1. Let $\left\{F_{u}: u \in V\right\}$ and $\left\{G_{u}: u \in V\right\}$ be the graph-directed selfsimilar sets generated by $\left\{V, E,\left\{f_{e}: e \in E\right\}\right\}$ and $\left\{V, E,\left\{g_{e}: e \in E\right\}\right\}$, respectively. Suppose that for each $e \in E$ the similitudes $f_{e}$ and $g_{e}$ have the same ratio $\rho_{e}$, and both $\left\{F_{u}: u \in V\right\}$ and $\left\{G_{u}: u \in V\right\}$ satisfy the strong separation condition. Then for each $u \in V$, we have $F_{u} \simeq G_{u}$.

Proof. Fix a $u \in V$. We denote by $E_{v}$ the set of directed edges starting from $v$ for $v \in V$. For a directed edge $e \in E$ we denote its initial and ending points by $e^{-}$ and $e^{+}$, respectively. Let

$$
c_{*}=\min _{v \in V} \min \left\{d\left(f_{e_{*}}\left(F_{e_{*}^{+}}\right), f_{e_{* *}}\left(F_{e_{* *}^{+}}\right)\right), d\left(g_{e_{*}}\left(G_{e_{*}^{+}}\right), g_{e_{* *}}\left(G_{e_{* *}^{+}}\right)\right): e_{*} \neq e_{* *} \in E_{v}\right\}
$$

and

$$
c^{*}=\max \left\{\text { diameter of the set } \bigcup_{v \in V} F_{v} \text {, diameter of the set } \bigcup_{v \in V} G_{v}\right\} \text {. }
$$


Then $c_{*}, c^{*}>0$. An infinite sequence of directed edges $e_{1} e_{2} \cdots$ is called admissible if $e_{i}^{+}$coincides with $e_{i+1}^{-}$for all $i \in \mathbf{N}$. Let

$$
\Sigma_{u}=\left\{e_{1} e_{2} \cdots: e_{1} e_{2} \cdots \text { is admissible with } e_{1}^{-}=u\right\} .
$$

Then the maps

$$
\Pi_{F}\left(e_{1} e_{2} \cdots\right)=\bigcap_{i=1}^{\infty} f_{e_{1}} \circ \cdots \circ f_{e_{i}}\left(F_{e_{i}^{+}}\right) \text {and } \Pi_{G}\left(e_{1} e_{2} \cdots\right)=\bigcap_{i=1}^{\infty} g_{e_{1}} \circ \cdots \circ g_{e_{i}}\left(G_{e_{i}^{+}}\right)
$$

are bijections between $\Sigma_{u}$ and $F_{u}$, and between $\Sigma_{u}$ and $G_{u}$ respectively. We shall check the bijection $\Pi_{G} \circ \Pi_{F}^{-1}$ is bi-Lipschitz. Let $x, y \in F_{u}$ with $x \neq y$. Then there exist unique $\left(e_{i}\right),\left(s_{i}\right) \in \Sigma_{u}$ such that $x=\Pi_{F}\left(e_{1} e_{2} \cdots\right), y=\Pi_{F}\left(s_{1} s_{2} \cdots\right)$. Let $\ell$ be the smallest integer such that $e_{\ell} \neq s_{\ell}$. Then we have $e_{\ell}^{-}=s_{\ell}^{-}$and $e_{\ell}^{+} \neq s_{\ell}^{+}$because of $e_{1}^{-}=s_{1}^{-}=u$. This implies that $x=f_{e_{1}} \circ \cdots \circ f_{e_{\ell-1}}\left(x^{*}\right)$ and $y=f_{e_{1}} \circ \cdots \circ f_{e_{\ell-1}}\left(y^{*}\right)$ with $x^{*} \in f_{e_{\ell}}\left(F_{e_{\ell}^{+}}\right), y^{*} \in f_{s_{\ell}}\left(F_{s_{\ell}^{+}}\right)$. So

$$
c_{*} \prod_{i=1}^{\ell-1} \rho_{e_{i}} \leq|x-y| \leq c^{*} \prod_{i=1}^{\ell-1} \rho_{e_{i}} .
$$

Note that

$$
\Pi_{G} \circ \Pi_{F}^{-1}(x)=\Pi_{G}\left(e_{1} e_{2} \cdots\right) \text { and } \Pi_{G} \circ \Pi_{F}^{-1}(y)=\Pi_{G}\left(s_{1} s_{2} \cdots\right),
$$

which implies, by the same argument as above, that

$$
c_{*} \prod_{i=1}^{\ell-1} \rho_{e_{i}} \leq\left|\Pi_{G} \circ \Pi_{F}^{-1}(x)-\Pi_{G} \circ \Pi_{F}^{-1}(y)\right| \leq c^{*} \prod_{i=1}^{\ell-1} \rho_{e_{i}} .
$$

Therefore, $\Pi_{G} \circ \Pi_{F}^{-1}$ is bi-Lipschitz.

Now we are ready to prove Theorem 1.1.

Proof of Theorem 1.1. By $\left\{f_{i}: 1 \leq i \leq m\right\}$ and $\left\{g_{i}: 1 \leq i \leq m\right\}$ we denote the iterated function systems corresponding to translations $\mathbf{a}=\left(a_{1}, a_{2}, \cdots, a_{m}\right)$ and $\mathbf{b}=\left(b_{1}, b_{2}, \cdots, b_{m}\right)$, respectively.

Without loss of generality we may assume that $f_{m}([0,1]) \cap f_{j}([0,1])=\emptyset$ for all $j<$ $m$, and that $g_{m}([0,1]) \cap g_{j}([0,1])=\emptyset$ for all $j<m$ in condition (III). To understand it, one only needs to notice the following facts: we have that $K_{\mathbf{c}}=1-K_{\mathbf{a}} \simeq K_{\mathbf{a}}$ for the translation $\mathbf{c}=\left(1-\lambda-a_{m}, 1-\lambda-a_{m-1}, \cdots, 1-\lambda-a_{2}, 1-\lambda-a_{1}\right) \in \mathbf{A}_{k_{1}, \cdots, k_{n}}$, and $\# \gamma_{\ell}(\mathbf{c})=\# \gamma_{\ell}(\mathbf{a})$ for $1 \leq \ell \leq n$. Let

$$
\gamma_{n+1}(\mathbf{a})=\{1, \cdots, m-1\} \backslash \bigcup_{\ell=1}^{n} \gamma_{\ell}(\mathbf{a})
$$

We relabel the elements of $\gamma_{\ell}(\mathbf{a})$ in its increasing order by digits $\left\{1+\sum_{j=1}^{\ell-1} \# \gamma_{j}(\mathbf{a}), 2+\right.$ $\left.\sum_{j=1}^{\ell-1} \# \gamma_{j}(\mathbf{a}), \cdots, \sum_{j=1}^{\ell} \# \gamma_{j}(\mathbf{a})\right\}$ with $\sum_{j=1}^{0} \# \gamma_{j}(\mathbf{a})=0$ and $1 \leq \ell \leq n+1$. By $h(\cdot)$ we denote this relabeling. Thus $h(j) \in\left\{1+\sum_{j=1}^{\ell-1} \# \gamma_{j}(\mathbf{a}), 2+\sum_{j=1}^{\ell-1} \# \gamma_{j}(\mathbf{a}), \cdots\right.$, $\left.\sum_{j=1}^{\ell} \# \gamma_{j}(\mathbf{a})\right\}$ for $j \in \gamma_{\ell}(\mathbf{a})$.

We partition $K_{\mathbf{a}}$ into $m+k_{1}-2$ pairwise disjoint nonempty compact sets, denoted by $K_{1}, \cdots, K_{m+k_{1}-2}$. The first $m-1$ members of them are defined by

$$
K_{h(j)}= \begin{cases}f_{j}\left(K_{\mathbf{a}}\right) \backslash f_{j} \circ f_{m}^{k_{\ell}-1}\left(K_{\mathbf{a}}\right) & \text { for } j \in \gamma_{\ell}(\mathbf{a}), 1 \leq \ell \leq n \\ f_{j}\left(K_{\mathbf{a}}\right) & \text { for } j \in \gamma_{n+1}(\mathbf{a}) .\end{cases}
$$


The later $k_{1}-1$ members of $K_{i}$ 's are defined by

$$
\left\{\begin{array}{l}
K_{m+k_{1}-2}=f_{m}^{k_{1}-1}\left(K_{\mathbf{a}}\right), \\
K_{m+t}=f_{m}^{t+1}\left(K_{\mathbf{a}}\right) \backslash f_{m}^{t+2}\left(K_{\mathbf{a}}\right) \quad \text { for } 0 \leq t<k_{1}-2 .
\end{array}\right.
$$

From (2) and (3) it follows that $K_{\mathbf{a}}=\bigcup_{i=1}^{m+k_{1}-2} K_{i}$ with disjoint union. It is important to notice that for $1 \leq \ell \leq n$

$f_{m}\left(K_{\mathbf{a}}\right)= \begin{cases}K_{m} \cup K_{m+1} \cup \cdots \cup K_{m+k_{\ell}-3} \cup f_{m}^{k_{\ell}-1}\left(K_{\mathbf{a}}\right) & \text { with disjoint union for } k_{\ell} \geq 3, \\ f_{m}\left(K_{\mathbf{a}}\right) & \text { for } k_{\ell}=2 .\end{cases}$

Thus we have for $j \in \gamma_{\ell}(\mathbf{a})$ with $1 \leq \ell \leq n$

$$
\begin{aligned}
K_{h(j)} & =f_{j}\left(K_{\mathbf{a}}\right) \backslash f_{j} \circ f_{m}^{k_{\ell}-1}\left(K_{\mathbf{a}}\right) \\
& =\left(f_{j}\left(K_{1} \cup \cdots \cup K_{m-1} \cup f_{m}\left(K_{\mathbf{a}}\right)\right)\right) \backslash f_{j} \circ f_{m}^{k_{\ell}-1}\left(K_{\mathbf{a}}\right)=\bigcup_{i=1}^{m+k_{\ell}-3} f_{j}\left(K_{i}\right) .
\end{aligned}
$$

It is obvious that for $j \in \gamma_{n+1}(\mathbf{a})$

$$
K_{h(j)}=\bigcup_{i=1}^{m+k_{1}-2} f_{j}\left(K_{i}\right)
$$

Finally, Note that $K_{\mathbf{a}}=K_{1} \cup \cdots \cup K_{m-1} \cup f_{m}\left(K_{\mathbf{a}}\right)$ with disjoint union. Thus, for $0 \leq t<k_{1}-2$

$$
K_{m+t}=f_{m}^{t+1}\left(K_{\mathbf{a}}\right) \backslash f_{m}^{t+2}\left(K_{\mathbf{a}}\right)=\bigcup_{i=1}^{m-1} f_{m}^{t+1}\left(K_{i}\right)
$$

and

$$
K_{m+k_{1}-2}= \begin{cases}f_{m}\left(K_{m+k_{1}-3}\right) \cup f_{m}\left(K_{m+k_{1}-2}\right) & \text { when } k_{1} \geq 3 \\ \bigcup_{i=1}^{m} f_{m}\left(K_{i}\right) & \text { when } k_{1}=2\end{cases}
$$

Therefore, $\left(K_{1}, \cdots, K_{m+k_{1}-2}\right)$ are graph-directed self-similar sets satisfying the strong separation condition. By the same argument as above by replacing $f_{i}$ by $g_{i}$, one can get pairwise disjoint nonempty compacts $K_{i}^{*}$ s with $K_{\mathbf{b}}=\bigcup_{1 \leq i \leq m+k_{1}-2} K_{i}^{*}$. The $\left(K_{1}^{*}, \cdots, K_{m+k_{1}-2}^{*}\right)$ are graph-directed self-similar sets satisfying the strong separation condition and obey the same equations as $K_{i}$ s with replacing $f_{i}$ by $g_{i}$. Thus $K_{i} \simeq K_{i}^{*}$ for $1 \leq i \leq m+k_{1}-2$, and so $K_{\mathbf{a}} \simeq K_{\mathbf{b}}$ because of the disjointness of $K_{i} \mathrm{~s}$ and disjointness of $K_{i}^{*}$ s.

Example 2.2. Let $0<\lambda<5^{-1}$. Take $\mathbf{a}=(0, \lambda(1-\lambda), 2 \lambda(1-\lambda), 3 \lambda, 1-\lambda)$ and $\mathbf{b}=\left(0, \lambda(1-\lambda), 2 \lambda, 3 \lambda-\lambda^{2}, 1-\lambda\right)$. Then one can check that $\mathbf{a}, \mathbf{b} \in \mathbf{A}_{2}, \gamma_{1}(\mathbf{a})=\{1,2\}$ and $\gamma_{1}(\mathbf{b})=\{1,3\}$. Thus $K_{\mathbf{a}} \simeq K_{\mathbf{b}}$ by Theorem 1.1.

The approach presented in this paper can be also applied for higher dimensional case.

Example 2.3. Let $0<\lambda<(2-\sqrt{2}) / 2$. Consider two IFSs $\left\{f_{i}: 1 \leq i \leq 6\right\}$ and $\left\{g_{i}: 1 \leq i \leq 6\right\}$ where

$$
\begin{array}{ll}
f_{1}(x, y)=\lambda(x, y), & f_{2}(x, y)=\lambda(x, y)+(1-\lambda, 0), \\
f_{3}(x, y)=\lambda(x, y)+(1-\lambda, 1-\lambda), & f_{4}(x, y)=\lambda(x, y)+(0,1-\lambda), \\
f_{5}(x, y)=\lambda(x, y)+\left(\lambda(1-\lambda),(1-\lambda)^{2}\right), & f_{6}(x, y)=\lambda(x, y)+(0,(1-\lambda)(1-2 \lambda)),
\end{array}
$$


and $g_{6}(x, y)=\lambda(x, y)+(\lambda(1-\lambda), \lambda(1-\lambda))$ with $g_{i}(x, y)=f_{i}(x, y)$ for $1 \leq i \leq 5$. Let $F$ and $G$ be the self-similar sets generated by IFSs $\left\{f_{i}: 1 \leq i \leq 6\right\}$ and $\left\{g_{i}: 1 \leq i \leq 6\right\}$, respectively. Then $F \simeq G$.

Proof. Figure 1 shows locations of squares $f_{i}\left([0,1]^{2}\right), 1 \leq i \leq 6$ and squares $g_{i}\left([0,1]^{2}\right), 1 \leq i \leq 6$. Let $F_{i}=f_{i}(F)$ for $i=1,2,3,5, F_{4}=f_{4}(F) \backslash f_{4} \circ f_{2}(F)$ and $F_{6}=f_{6}(F) \backslash f_{6} \circ f_{3}(F)$. Then $F_{i}, 1 \leq i \leq 6$, are pairwise disjoint nonempty compact sets such that $F=\bigcup_{1 \leq i \leq 6} F_{i}$ since $f_{4} \circ f_{2}=f_{5} \circ f_{4}$ and $f_{6} \circ f_{3}=f_{5} \circ f_{1}$.

Thus we have

$$
\left\{\begin{array}{l}
F_{i}=f_{i}\left(F_{1}\right) \cup f_{i}\left(F_{2}\right) \cup f_{i}\left(F_{3}\right) \cup f_{i}\left(F_{4}\right) \cup f_{i}\left(F_{5}\right) \cup f_{i}\left(F_{6}\right) \quad \text { for } i=1,2,3,5, \\
F_{4}=f_{4}\left(F_{1}\right) \cup f_{4}\left(F_{3}\right) \cup f_{4}\left(F_{4}\right) \cup f_{4}\left(F_{5}\right) \cup f_{4}\left(F_{6}\right), \\
F_{6}=f_{6}\left(F_{1}\right) \cup f_{6}\left(F_{2}\right) \cup f_{6}\left(F_{4}\right) \cup f_{6}\left(F_{5}\right) \cup f_{6}\left(F_{6}\right),
\end{array}\right.
$$
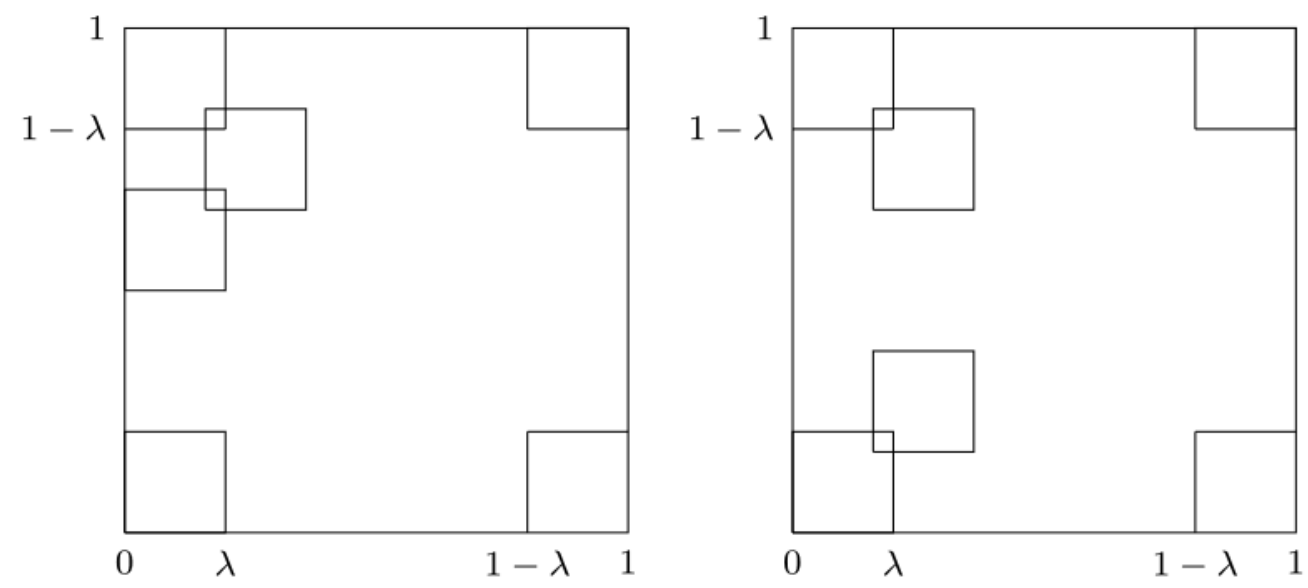

Figure 1. Squares $f_{i}\left([0,1]^{2}\right)$ on the left side and squares $g_{i}\left([0,1]^{2}\right)$ on the right side.

By the same way as above let $G_{1}=g_{5}(G), G_{2}=g_{2}(G), G_{3}=g_{3}(G), G_{4}=$ $g_{4}(G) \backslash g_{4} \circ g_{2}(G), G_{5}=g_{6}(G)$ and $G_{6}=g_{1}(G) \backslash g_{1} \circ g_{3}(G)$. We have $G_{i}, 1 \leq i \leq 6$, are pairwise disjoint nonempty compact sets with $G=\bigcup_{1 \leq i \leq 6} G_{i}$ and satisfy

$$
\left\{\begin{array}{l}
G_{1}=g_{5}\left(G_{1}\right) \cup g_{5}\left(G_{2}\right) \cup g_{5}\left(G_{3}\right) \cup g_{5}\left(G_{4}\right) \cup g_{5}\left(G_{5}\right) \cup g_{5}\left(G_{6}\right), \\
G_{2}=g_{2}\left(G_{1}\right) \cup g_{2}\left(G_{2}\right) \cup g_{2}\left(G_{3}\right) \cup g_{2}\left(G_{4}\right) \cup g_{2}\left(G_{5}\right) \cup g_{2}\left(G_{6}\right), \\
G_{3}=g_{3}\left(G_{1}\right) \cup g_{3}\left(G_{2}\right) \cup g_{3}\left(G_{3}\right) \cup g_{3}\left(G_{4}\right) \cup g_{3}\left(G_{5}\right) \cup g_{3}\left(G_{6}\right), \\
G_{5}=g_{6}\left(G_{1}\right) \cup g_{6}\left(G_{2}\right) \cup g_{6}\left(G_{3}\right) \cup g_{6}\left(G_{4}\right) \cup g_{6}\left(G_{5}\right) \cup g_{6}\left(G_{6}\right), \\
G_{4}=g_{4}\left(G_{1}\right) \cup g_{4}\left(G_{3}\right) \cup g_{4}\left(G_{4}\right) \cup g_{4}\left(G_{5}\right) \cup g_{4}\left(G_{6}\right), \\
G_{6}=g_{1}\left(G_{1}\right) \cup g_{1}\left(G_{2}\right) \cup g_{1}\left(G_{4}\right) \cup g_{1}\left(G_{5}\right) \cup g_{1}\left(G_{6}\right) .
\end{array}\right.
$$

Thus $F \simeq G$ by Lemma 2.1 .

Example 2.4. Let $0<\lambda<\frac{1}{7}$. Let $G$ be the self-similar set generated by the IFS $\left\{g_{i}: 1 \leq i \leq 6\right\}$ given in Example 2.3. Let $F$ be the self-similar set generated by the IFS $\left\{f_{i}: 1 \leq i \leq 6\right\}$ where $f_{1}(x)=\lambda x, f_{2}(x)=\lambda x+2 \lambda, f_{3}(x)=\lambda x+3 \lambda-\lambda^{2}$, $f_{4}(x)=\lambda x+4 \lambda-2 \lambda^{2}, f_{5}(x)=\lambda x+5 \lambda$ and $f_{6}(x)=\lambda x+1-\lambda$. Then $F \simeq G$.

Proof. Note that $F^{*}=F \times\{0\}$ is the self-similar set in $\mathbf{R}^{2}$ generated by the IFS:

$$
\begin{array}{ll}
f_{1}^{*}(x, y)=\lambda(x, y), & f_{2}^{*}(x, y)=\lambda(x, y)+(2 \lambda, 0), \\
f_{3}^{*}(x, y)=\lambda(x, y)+\left(3 \lambda-\lambda^{2}, 0\right), & f_{4}^{*}(x, y)=\lambda(x, y)+\left(4 \lambda-2 \lambda^{2}, 0\right), \\
f_{5}^{*}(x, y)=\lambda(x, y)+(5 \lambda, 0), & f_{6}^{*}(x, y)=\lambda(x, y)+(1-\lambda, 0) .
\end{array}
$$


By letting $F_{1}=f_{3}^{*}\left(F^{*}\right), F_{2}=f_{1}^{*}\left(F^{*}\right), F_{3}=f_{6}^{*}\left(F^{*}\right), F_{5}=f_{5}^{*}\left(F^{*}\right), F_{6}=f_{2}^{*}\left(F^{*}\right) \backslash$ $f_{2}^{*} \circ f_{6}^{*}\left(F^{*}\right), F_{4}=f_{4}^{*}\left(F^{*}\right) \backslash f_{4}^{*} \circ f_{1}^{*}\left(F^{*}\right)$, one can get $F^{*}$ has the same graph-directed structure as $G$. Thus we have $G \simeq F^{*} \simeq F$ by Lemma 2.1.

Remark 2.5. It is natural to compare our method with the idea used in [11]. On the one hand, our method cannot prove the Lipschitz equivalence between the $\{1,4,5\}$-Cantor set and the $\{1,3,5\}$-Cantor set. The main difficulty, which is crucial, is that our idea only transforms the $\{1,4,5\}$-Cantor set into a graph-directed selfsimilar sets with the open set condition rather than the strong separation condition. It is not enough if we only obtain the open set condition. That is why we cannot reprove the main result of [11]. On the other hand, in terms of the approach of [11], it seems that we cannot obtain Theorem 1.1. In brief, these two methods above are independent, i.e. the idea of [11] is useful when one tackles the self-similar sets with the open set condition, while our method is effective for the self-similar sets with exact overlaps.

Acknowledgments. The first author was granted by the China Scholarship Council No. 201606140059. The second author was granted by the China Scholarship Council No. 201206140003. The third author was supported by NSFC Nos. 11271137, 11571144, 11671147 and Science and Technology Commission of Shanghai Municipality (STCSM), grant No. 13dz2260400.

\section{References}

[1] Dajani, K., and K. JiAng: Subshift of finite type and self-similar sets. - arXiv:1509.04900, 2015.

[2] David, G., and S. Semmes: Fractured fractals and broken dreams: self-similar geometry through metric and measure. - Oxford Lecture Ser. Math. Appl. 7, Clarendon Press, 1997.

[3] Falconer, K. J., and D. T. MARsh: Classification of quasi-circles by Hausdorff dimension. Nonlinearity 2, 1989, 489-493.

[4] Falconer, K. J., and D. T. MArsh: On the Lipschitz equivalence of Cantor sets. - Mathematika 39, 1992, 223-233.

[5] Guo, Q., H. Li, Q. WAnG, and L. XI: Lipschitz equivalence of a class of self-similar sets with complete overlaps. - Ann. Acad. Sci. Fenn. Math. 37, 2012, 229-243.

[6] Hutchinson, J. E.: Fractals and self-similarity. - Indiana Univ. Math. J. 30, 1981, 713-747.

[7] Li, B. M., W. X. Li, and J. J. MiaO: Lipschitz equivalence of McMullen sets. - Fractals 21, 2013, 1-11.

[8] LuO, J. J., and K.-S. LAU: Lipschitz equivalence of self-similar sets and hyperbolic boundaries. - Adv. Math. 235, 2013, 555-579.

[9] Mattila, P., and P. SaAranen: Ahlfors-David regular sets and bilipschitz maps. - Ann. Acad. Sci. Fenn. Math. 34, 2009, 487-502.

[10] Mauldin, R. D., and S. C. Williams: Hausdorff dimension in graph directed constructions. - Trans. Amer. Math. Soc. 309, 1988, 811-829.

[11] RaO, H., H.-J. RuAn, and L.-F. Xi: Lipschitz equivalence of self-similar sets. - C. R. Math. Acad. Sci. Paris 342, 2006, 191-196.

[12] RAO, H., H. RuAn, and Y. WANG: Lipschitz equivlance of Cantor sets and algebraic properties of contraction ratios. - Trans. Amer. Math. Soc. 364, 2012, 1109-1126.

[13] RuAn, H.-J., Y. WANG, and L.-F. XI: Lipschitz equivalence of self-similar sets with touching structures. - Nonlinearity 27, 2014, 1299-1321. 
[14] Wen, Z.-X., Z.-Y. Zhu, and G.-T. Deng: Lipschitz equivalence of a class of general Sierpinski carpets. - J. Math. Anal. Appl. 385, 2012, 16-23.

[15] XI, L.-F., and Y. XIONG: Lipschitz equivalence of fractals generated by nested cubes. - Math. Z. $271,2012,1287-1308$.

[16] XI, L.-F., and Y. XIONG: Lipschitz equivalence class, ideal class and the gauss class number problem. - arXiv:1304.0103, 2013.

Received 4 November $2015 \bullet$ Accepted 2 November 2016 